\title{
Giant renal angiomyolipoma: A case report
}

\author{
PEIJIE CHEN ${ }^{1,2 *}$, LU JIN $^{1,3^{*}}$, YU YANG ${ }^{1}$, ZEBO CHEN $^{1}$, LIANGCHAO NI ${ }^{1}$, \\ SHANGQI YANG ${ }^{1}$ and YONGQING LAI ${ }^{1}$ \\ ${ }^{1}$ Department of Urology, Peking University Shenzhen Hospital, Shenzhen, Guangdong 518036;
${ }^{2}$ Department of Urology, Shantou University Medical College, Shantou, Guangdong 515041;
${ }^{3}$ Department of Urology, Anhui Medical University, Hefei, Anhui 230032, P.R. China
}

Received September 21, 2016; Accepted April 24, 2017

DOI: $10.3892 /$ mco.2017.1305

\begin{abstract}
Renal angiomyolipoma (AML), also referred to as renal hamartoma, is a rare solid tumor without malignant characteristics. The inheritance pattern of renal AML is autosomal dominant. If the lesion grows to a large size, a series of clinical manifestations and serious complications may occur. We herein present a case of giant renal AML in a 34-year-old female patient, who presented with left-sided abdominal bloating for $\sim 6$ months. Following abdominal ultrasound and computed tomography examination, the patient underwent total left nephrectomy. The resected mass was sized 29x20x10 cm. Postoperative histopathological examination confirmed the lesion as a giant renal AML. There are only few cases of giant renal AML $(>20 \mathrm{~cm})$ reported in the literature to date. Due to the large size of the tumor, it is crucial to report similar cases, their diagnosis and treatment.
\end{abstract}

\section{Introduction}

Renal angiomyolipoma (AML) is a rare neoplasm consisting of mature adipose tissue, thick-walled blood vessels and smooth muscle in different proportions. AML is also referred to as renal hamartoma. Previous studies reported that renal AML may grow by $4 \mathrm{~cm}$ each year in its maximum dimension $(1,2)$. When renal AMLs grow to a size of $>10 \mathrm{~cm}$, they are referred to as 'giant' AMLs. Giant renal AML is uncommonly reported in the literature (3). However, due to the hemorrhagic aneurysms that develop with the enlarging AML, the incidence of compression symptoms and the risk of bleeding from rupture increase (4). Due to the nature of this tumor, obvious clinical symptoms occur in the majority of giant renal AML cases, and

Correspondence to: Professor Yongqing Lai, Department of Urology, Peking University Shenzhen Hospital, 1120 Lianhua Road, Shenzhen, Guangdong 518036, P.R. China

E-mail: yqlord@163.com

*Contributed equally

Key words: renal angiomyolipoma, hamartomas, nephrectomy, renal tumor, giant kidney tumor protecting renal function is the main therapeutic target (5). We herein report the clinical course, diagnosis and treatment of a patient with a giant renal AML.

\section{Case report}

In May 2016, a 34-year-old female patient was admitted to the Department of Urology of Peking University Shenzhen Hospital (Shenzhen, China) with a 6-month history of progressive bloating sensation in the abdomen. On palpation, a sizeable mass was identified in the left lateral abdominal area. The patient underwent an ultrasound examination and a hyperechoic mass sized $20 \mathrm{~cm}$ was identified in the left abdominal area, possibly originating from the left kidney. Furthermore, computed tomography (CT) examination revealed a large mass with mixed density measuring $\sim 27 \times 22 \times 12 \mathrm{~cm}$. The lesion appeared to arise from the left kidney, displacing the kidney and intestines to the right (Fig. 1). There were no symptoms or radiological findings suggesting tuberous sclerosis complex (TSC). The adrenal glands bilaterally and the right kidney appeared to be normal, and the results of blood biochemistry tests were within the normal range. Following abdominal laparoscopic exploration, conversion to laparotomy was decided. The giant mass was found to originate from the left kidney. A left total nephrectomy was performed to remove the giant tumor and the non-functioning kidney. Postoperatively, supportive treatment was administered with fluid infusion and the patient's recovery was uneventful. On the date of the last follow-up (April 24, 2017), the patient remained disease-free.

The histopathological examination revealed that the lesion $(29 \times 20 \times 10 \mathrm{~cm})$ consisted of mature adipose tissue, thick-walled blood vessels and smooth muscle in different proportions, and originated from the left kidney (Fig. 2). Immunohistochemical staining of the tumor revealed that the tumor cells were positive for smooth muscle actin, human melanoma black-45 (HMB-45), melan-A and S-100, and the $\mathrm{Ki}-67$ proliferation index was $1 \%$. Base on those findings, the patient was diagnosed with giant renal AML.

\section{Discussion}

Renal AML is a benign neoplasm arising from mesenchymal elements and was first reported in 1951 (6). AML is also referred to as 'hamartoma' due its varying composition, 


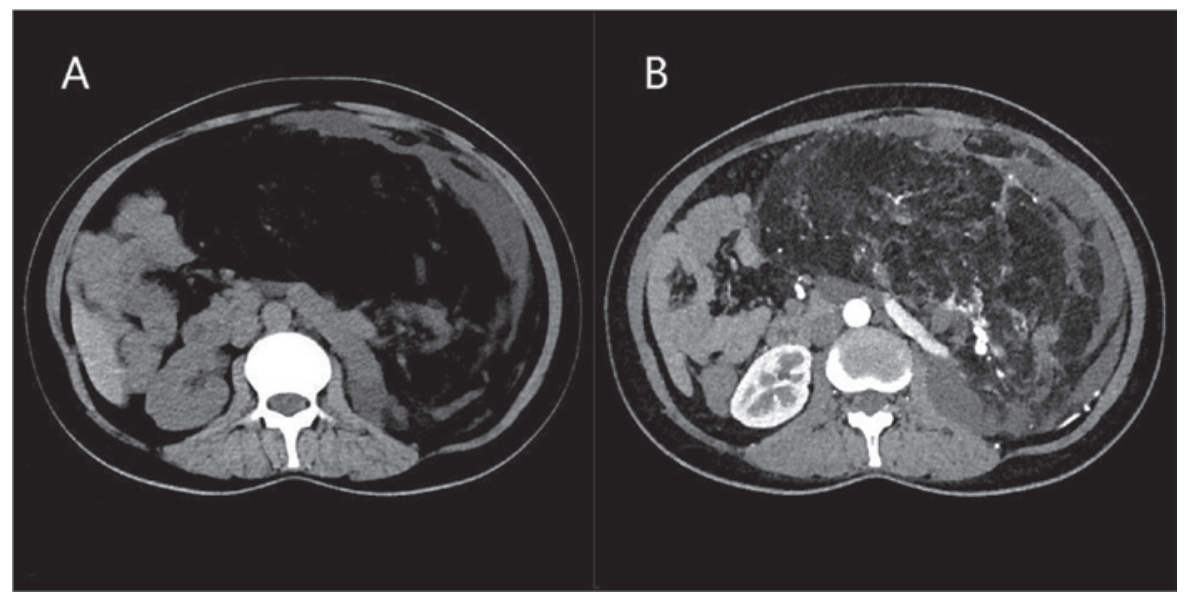

Figure 1. Computed tomography images of renal angiomyolipoma (A) prior to and (B) following intravenous administration of contrast material.

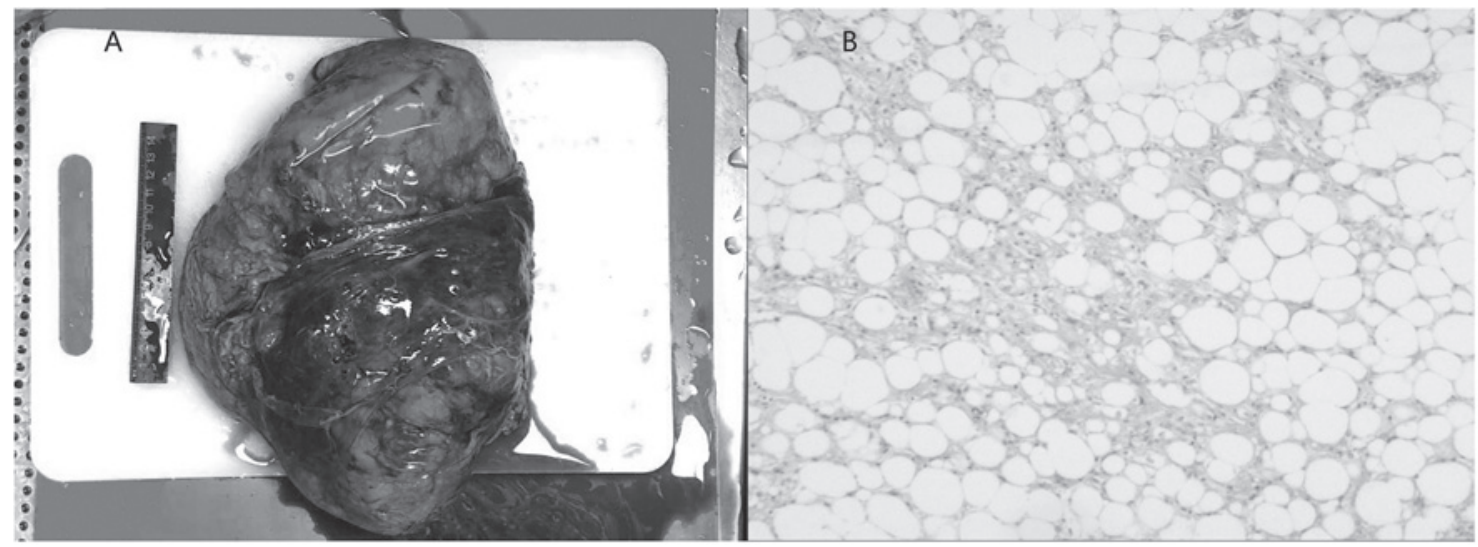

Figure 2. (A) Surgical specimen of a giant renal angiomyolipoma, measuring $29 \times 20 \times 10 \mathrm{~cm}$. (B) On histopathological examination, the tumor consisted of mature adipose tissue, thick-walled blood vessels and smooth muscle (hematoxylin and eosin staining; magnification, $\mathrm{x} 400$ ).

including adipose tissue, smooth muscle and blood vessels (7). These tumors are usually divided into two types, namely in the context of TSC or as sporadic; the case discussed herein belongs to the latter category. It was previously reported that renal AML may grow BY $4 \mathrm{~cm}$ each year in its maximum dimension $(1,2)$. When the diameter of renal AML reaches $>10 \mathrm{~cm}$, the tumor is referred to as 'giant'. Reports of giant renal AML in the literature are uncommon (3), with the largest renal AML $(39 \times 25 \mathrm{cx} 9 \mathrm{~cm})$ reported in 2013 by Taneja et al (8). This giant tumor is rich in thick-walled blood vessel that are prone to rupture and bleeding. Due to the hemorrhagic aneurysms that develop with the enlarging AML, the incidence of compression symptoms and the risk of bleeding from rupture increase (4). Lower back pain, hematuria and shock are the most important clinical manifestations suggesting the occurrence of retroperitoneal bleeding, referred to as Wunderlich's syndrome. These clinical manifestations are the main reason for renal AML patients seeking medical attention.

The majority of tumors sized $<4 \mathrm{~cm}$ are asymptomatic and patients may be managed conservatively (9), or treated by radiofrequency ablation (10). As the tumor grows, compression of the gastrointestinal tract may cause alimentary symptoms; in addition, the presence of a mass in the abdominal area may be easily confirmed by palpation. The mass appears to be echogenic on ultrasound and has the density of fat on CT scanning. When the diameter of renal AML becomes $>4 \mathrm{~cm}$, particularly for giant tumors with persistent hemorrhage or suspicion of malignancy, partial or total nephrectomy is the treatment of choice (11). In the present case, total nephrectomy was performed as the left kidney was non-functional. Histopathological examination of the renal AML revealed that the main component was normal/mature adipose (12). HMB-45 positivity on immunohistochemical staining may be used to distinguish renal AML from other renal tumors (13).

In conclusion, giant renal AML may be identified by imaging and the diagnosis is confirmed by histological and immunohistochemical examination. As this tumor is associated with a high risk of hemorrhage, partial or total nephrectomy, rather than conservative treatment, is the treatment of choice in order to reduce the risk of bleeding and relieve the compression symptoms caused by the giant mass.

\section{Acknowledgements}

The present study was supported by the National Natural Science Foundation of China (grant no. 81101922), the Science and Technology Development Fund Project of Shenzhen (grant 
nos. JCYJ20130402114702124 and JCYJ20150403091443329) and the fund of Guangdong Key Medical Subject.

\section{References}

1. Seyam RM, Bissada NK, Kattan SA, Mokhtar AA, Aslam M, Fahmy WE, Mourad WA, Binmahfouz AA, Alzahrani HM and Hanash KA: Changing trends in presentation, diagnosis and management of renal angiomyolipoma: Comparison of sporadic and tuberous sclerosis complex-associated forms. Urology 72 : 1077-1082, 2008.

2. Ewalt DH, Sheffield E, Sparagana SP, Delgado MR and Roach ES: Renal lesion growth in children with tuberous sclerosis complex. J Urol 160: 141-145, 1998.

3. Chronopoulos PN, Kaisidis GN, Vaiopoulos CK, Perits DM, Varvarousis MN, Malioris AV, Pazarli E and Skandalos IK: Spontaneous rupture of a giant renal angiomyolipoma-Wunderlich's syndrome: Report of a case. Int J Surg Case Rep 19: 140-143, 2016.

4. De Luca S, Terrone C and Rossetti SR: Management of renal angiomyolipoma: A report of 53 cases. BJU Int 83: 215-218, 1999.

5. Zhou Y, Tang Y, Tang J, Deng F, Gan YU and Dai Y: Total nephrectomy with nephron-sparing surgery for a giant bilateral renal angiomyolipoma: A case report. Oncol Lett 10: 2450-2452, 2015.

6. Morgan GS, Straumfjord JV and Hall EJ: Angiomyolipoma of the kidney. J Urol 65: 525-527, 1951.

7. Kulkarni B, Desai SB, Dave B, Tongaonkar HB, Kulkarni JN and Chinoy RF: Renal angiomyolipomas-a study of 18 cases. Indian J Pathol Microbiol 48: 459-463, 2005.

8. Taneja R and Singh DV: Giant renal angiomyolipoma: Unusua cause of huge abdominal mass. J Clin Imaging Sci 3: 56, 2013.
9. Bora A, Soni A, Sainani N and Patkar D: Emergency embolization of a bleeding renal angiomyolipoma using polyvinyl alcohol particles. Diagn Interv Radiol 13: 213-216, 2007.

10. Gregory SM, Anderson CJ and Patel U: Radiofrequency ablation of large renal angiomyolipoma: Median-term follow-up. Cardiovasc Intervent Radiol 36: 682-689, 2013.

11. Faddegon S and So A: Treatment of angiomyolipoma at a tertiary care centre: The decision between surgery and angioembolization. Can Urol Assoc J 5: E138-E141, 2011.

12. Serrano Frago P, Del Agua Arias Camisón C, Gil Sanz MJ, Allué López M, Gonzalvo Ibarra A, Plaza Mas L and Rioja Sanz LA: Controversies related to epithelioid variant of renal angiomyolipoma: A review of the literature. Urology 67: 846.e3-e5, 2006.

13. Vitaly M, Surena FM and Christopher GW: Campbell-Walsh, Urology. Benign renal tumors. 10th edition. Philadelphia, Saunders Elsevier, pp1498-pp501, 2012. 